\title{
Calcium hydroxide: An efficient and mild base for one-pot synthesis of curcumin and it's analogues
}

\author{
Pramod S. Kulkarni ${ }^{\mathrm{b}}$, Dasharath D. Kondhare ${ }^{\mathrm{b}}$, \\ Ravi Varala ${ }^{a}$, Pudukulathan K. Zubaidha ${ }^{\mathrm{b}}$ \\ ${ }^{a}$ Department of Chemistry, Rajiv Gandhi University of Knowledge Technologies, \\ Mudhole, Adilabad, Andhra Pradesh - 504 107, India \\ ${ }^{b}$ School of Chemical Sciences, Swami Ramanand Teerth Marathwada University, \\ Nanded, Maharashtra - 431 606, India \\ ravivarala@gmail.com
}

\begin{abstract}
Calcium hydroxide was found to be an efficient mild base for the one-pot synthesis of curcumin and its analogues obtained by condensation of one equivalent of acetyl acetone with two equivalents of corresponding aromatic aldehyde. The present protocol offers various advantages such as high yields, inexpensive easily available mild base, easy workup and eco-friendly method.
\end{abstract}

Keywords: Calcium hydroxide, acetyl acetone, substituted benzaldehydes, DMF, Curcumin

\section{Introduction}

Naturally derived products are the most valuable source for drugs and their lead compounds. Curcumin is such an interesting molecule from Mother Nature, commonly known as diferuloyl methane and is a dietary constituent of turmeric. It is a yellow, lipophilic polyphenol compound derived from the rhizome of the herb Curcuma longa $\mathrm{L}$.. Turmeric is commonly used as a colouring agent in cooking and is used as an herb in traditional indian and chinese medicine (Kuttan et al. 1985, 1987). Chemically, curcumin is a bis- $\alpha, \beta$-unsaturated $\beta$-diketone, which exhibits keto-enol tautomerism having a predominant keto form in acidic and neutral solutions and stable enol form in alkaline medium (Anand et al. 2008).

Curcumin possesses diverse pharmalogoical activities including anti-inflammatory, antioxidant, anticarinogenic (especially, colon cancer), treating alzheimer's disease, anti-HIV, antimicrobial, antidiabetic (Kumar et al. 2003, Jagetia et al. 2007, Mukherjee et al. 2007, Chakraborty et al. 2006) and for other disorders (Hsu et al. 2007). Therefore, curcumin is labeled as a multi-targeted drug and researchers call curcumin 'solid gold' for its curative properties. It has been postulated that the biological efficacy of curcumin arises from the electrophilic nature of its central $\beta$-diketone component (Furness et al. 2005). Recent clinical studies reported that curcumin could be orally administered up to $12 \mathrm{~g} /$ day without any toxic effect in humans (Liang et al. 2009). Very few methods exist for synthesis of curcumin and the first method developed by Paban (Paban et al. 1937) calls for preparation of carbomethoxyferuloic acid chloride involving tedious steps while the other method require condensation of carbomethoxyferuloyl chloride with vinyl acetate in the presence of anhydrous aluminum chloride in carbon disulfide (Tarabasanu et al. 1997).

Biosynthetic route for curcumin in plant cells follow blocking of the active methylene group via enol phosphoric acid and this strategy was successfully extended by Jan Van Alphen and Hendrik Jacob Paban for the synthesis of curcumin in 1959. In this method, boric anhydride was used to block the medial methylene group of acetyl acetone and condensation of terminal methyl groups with vanillin was effected under basic conditions (Bratu 2004). Microwave assisted-boron oxide and microwave assisted-CaO curcumin synthesis are also documented (Nichols et al. 2006 and Elavarasan et al. 2012). However these reported methods suffer from drawbacks such as harsh reaction condition, tedious workup procedure, low yield, limitations for scale-up, expensive catalyst and isolation of product requires extraction process. Keeping in view of the limited methods reported and conditions employed, we have developed a very facile protocol for synthesis of curcumin and its analogues using calcium hydroxide for the first time as a base for condensation of acetyl acetone with various substituted benzaldehydes. Calcium hydroxide is a mild water soluble base with high $\mathrm{pH}$ in water (12.6), obtained through calcination of calcium carbonate (Greenwood et al. 1984).

Use of calcium hydroxide in organic synthesis is very rare and some of its applications include its use in selective C-4 acylation of pyrazolones (Hari 
et al. 2011), transesterification of oil (Chen et al. 2011) and synthesis of benzopyrans (Saimoto et al. 1996, Jensen 1959). In continuation of our interest in developing novel synthetic methodologies, particularly carbon-carbon, carbon-heteroatom bond formations (Narayana et al. 2012), herein we report our brief findings for a highly efficient method for the synthesis of Curcumin via $\mathrm{Ca}(\mathrm{OH})_{2}$-mediated condensation (Scheme 1).

\section{Results and Discussion}

Acetyl acetone contains two terminal methyl groups and one central methylene group. The central methylene group is more reactive than terminal methyl groups. Therefore to effect condensation at the terminal methyl groups it is necessary to block active methylene group throughout the synthetic process and deblock the same at the end of the synthesis. Acetyl acetone exists in keto and enol forms. The enol form coordinates through both oxygen atoms with the loss of a proton to form a chelate ring and thus acetylacetone acts as a bidentate ligand. Calcium is divalent in nature and it has tendency to form chelate with bidentate ligand. Using this logic it was anticipated that calcium would block the active methylene group via chelation and also act as a base to bring about condensation at the terminal ends with benzaldehyde in one pot. In the initial experiment, we stirred 2 equivalent of vanillin, 1 equivalent of acetyl acetone and 3 equivalent of calcium hydroxide in methanol at $50-60{ }^{\circ} \mathrm{C}$. The progress of the reaction was monitored by TLC and after $48 \mathrm{~h}$, we observed the consumption of starting materials and formation of new spot. After work-up and purification, the product was characterized by spectroscopic methods and confirmed to be curcumin (Table 2, $3 \mathrm{~m}, 50 \%$ yield).

IR spectrum of curcumin showed a strong and broad hydroxyl absorption band at $3468.5 \mathrm{~cm}^{-1}$ and the ${ }^{1} \mathrm{H}$ NMR spectrum of curcumin contains two singlets at 3.84 and $9.74 \mathrm{ppm}$ due to the protons of the two methoxy groups and the protons of the two hydroxyl groups respectively, which reflects its symmetric structure. ${ }^{13} \mathrm{C}$ NMR spectral data show that curcumin exists primarily in the enol form and not as the diketone.

Tab. 1. $\mathrm{Ca}(\mathrm{OH})_{2}$-mediated synthesis of curcumin in different solvents.

\begin{tabular}{cccc}
\hline Entry & Solvent & Time (h) & Yield (\%) \\
\hline 1 & Ethanol & $46-48$ & 52 \\
2 & Methanol & $48-50$ & 50 \\
3 & Acetonitrile & $46-48$ & 62 \\
4 & DMF & 16 & 82 \\
\hline
\end{tabular}

${ }^{\mathrm{a}}$ Isolated yield.

Having successfully obtained curcumin using calcium hydroxide, next we focused out attention on improving the yield and reaction time. The reaction was studied in different solvents and the results are summarized in Table 1. As is obvious from the Table 1 the reaction proceeded well in DMF at $110{ }^{\circ} \mathrm{C}$ (entry 4 , Table 1 ) in terms of both yield and reaction time, compared to other solvents. Work up involves pouring of the reaction mixture in to cold water and isolation of the product by simple filtration. The scope of this protocol for tolerance of functional groups and steric factor was investigated by subjecting different substituted benzaldehydes to the optimized reaction conditions and the results are presented in Table 2.

It is worth mentioning that aromatic aldehydes bearing both electron donating and withdrawing groups underwent facile condensation to afford the corresponding curcumin analogues in good yield. While study on the effect of substitution on aromatic ring reveals that the reaction is prone to steric hinderance and yields suffer in case of ortho substituted aldehydes. In general, the first step was the reaction of acetylacetone with $\mathrm{Ca}(\mathrm{OH})_{2}$ building a Ca complex, which inhibited an unpleased Knoevenagel reaction. After addition of a corresponding benzaldehyde, the condensation of the acetylacetone-Ca complex with the aldehyde and an additional elimination occurred. Workup using cold dil. HCl cleaved the Ca complex to give the desired curcumin analogues.<smiles>[R]c1ccc(/C=C/C(=O)/C=C(O)/C=C/c2ccc([R3])c([R])c2[R])c([R])c1[R]</smiles>

Scheme 1. Calcium hydroxide-mediated synthesis of curcumin. 
Tab. 2. $\mathrm{Ca}(\mathrm{OH})_{2}$-mediated synthesis of Curcumin from substituted benzaldehydes.

\begin{tabular}{|c|c|c|c|c|c|c|}
\hline Entry & $\mathbf{R}^{1}$ & $\mathbf{R}^{2}$ & $\mathbf{R}^{3}$ & Product (3) & Yield $^{\mathrm{a}}(\%)$ & Melting Point $\left({ }^{\circ} \mathrm{C}\right)$ \\
\hline 1 & $\mathrm{H}$ & $\mathrm{H}$ & $\mathrm{H}$ & $3 \mathrm{a}$ & 86 & 142 Nurfina et al. \\
\hline 2 & $\mathrm{H}$ & $\mathrm{H}$ & $\mathrm{CH}_{3}$ & $3 b$ & 78 & $111-113$ Nurfina et al. \\
\hline 3 & $\mathrm{H}$ & $\mathrm{H}$ & $\mathrm{Cl}$ & $3 c$ & 83 & $151-153$ Nurfina et al. \\
\hline 4 & $\mathrm{Cl}$ & $\mathrm{H}$ & $\mathrm{H}$ & $3 d$ & 75 & $116-119$ \\
\hline 5 & $\mathrm{H}$ & $\mathrm{H}$ & $\mathrm{OCH}_{3}$ & $3 \mathrm{e}$ & 78 & $161-162$ Nurfina et al. \\
\hline 6 & $\mathrm{OH}$ & $\mathrm{H}$ & $\mathrm{H}$ & $3 \mathrm{f}$ & 69 & 187 Babua et al. \\
\hline 7 & $\mathrm{H}$ & $\mathrm{OCH}_{3}$ & $\mathrm{OCH}_{3}$ & $3 g$ & 77 & $129-130$ Nurfina et al. \\
\hline 8 & $\mathrm{H}$ & $\mathrm{H}$ & $\mathrm{NO}_{2}$ & $3 \mathrm{~h}$ & 88 & $146-147$ Bratu et al. 2005 \\
\hline 9 & $\mathrm{H}$ & $\mathrm{NO}_{2}$ & $\mathrm{H}$ & $3 \mathrm{i}$ & 89 & 161-163 Hahm et al. \\
\hline 10 & $\mathrm{NO}_{2}$ & $\mathrm{H}$ & $\mathrm{H}$ & $3 \mathrm{j}$ & 74 & $141-142$ Hahm et al. \\
\hline 11 & $\mathrm{H}$ & $\mathrm{H}$ & $\mathrm{N}\left(\mathrm{CH}_{3}\right)_{2}$ & $3 \mathrm{k}$ & 67 & $147-149$ Bratu et al. 2005 \\
\hline 12 & $\mathrm{H}$ & $\mathrm{H}$ & $\mathrm{Br}$ & 31 & 80 & $154-156$ \\
\hline 13 & $\mathrm{H}$ & $\mathrm{OCH}_{3}$ & $\mathrm{OH}$ & $3 \mathrm{~m}$ & 82 & $181-182$ Nurfina et al. \\
\hline 14 & $\mathrm{H}$ & $\mathrm{H}$ & $\mathrm{OH}$ & $3 n$ & 72 & 175 Nurfina et al. \\
\hline 15 & $\mathrm{OCH}_{3}$ & $\mathrm{H}$ & $\mathrm{H}$ & 30 & 68 & $122-124$ Nurfina et al. \\
\hline 16 & & - & - & $3 p$ & 76 & $185-187$ \\
\hline
\end{tabular}

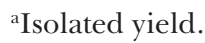

\section{Experimental section}

All purchased chemicals were of analytical grade and used without further purification. All the yields were calculated after purification of products by crystallization process. Melting point is determined by open capillary method and uncorrected. IR spectra were recorded on a Perkin-Elmer FTIR-1710 spectrophotometer. The NMR spectra were obtained on a Bruker DRX-300 Advance instrument using $\mathrm{CDCl}_{3}$ as solvent and TMS as internal standard. All products were characterized by melting point, IR, ${ }^{1} \mathrm{HNMR}{ }^{13} \mathrm{C}$ NMR and mass spectra.

\section{General Procedure for synthesis of curcumin analogues ( $3 a-p)$ :}

To a mixture of 2 equivalent of substituted benzaldehyde (10 mmol), 1 equivalent of acetylacetone $(5 \mathrm{mmol})$ dissolved in $20 \mathrm{~mL}$ of DMF was added 3 equivalents of calcium hydroxide $(15 \mathrm{mmol})$, and the reaction mixture was stirred at $110^{\circ} \mathrm{C}$. The progress of the reaction was monitored by TLC. After completion of the reaction, it was poured into cold water, neutralized with cold dil. HCl up to $\mathrm{pH}$ 4 to 5 . The solid precipitated was filtered, washed with cold water and purified by crystallization from ethanol.

\section{Spectral data for Curcumin (1,7-Bis(4-hydroxy- 3-methoxyphenyl)-1,6-heptadiene-3,5-dione, 3m)}<smiles>COc1cc(/C=C/C(=O)/C=C(O)/C=C/c2ccc(O)c(OC)c2)ccc1O</smiles>

m.p. $=181-182^{\circ} \mathrm{C}$

IR (KBr): v 3468 (br s, OH), $1627(\mathrm{C}=\mathrm{O}), 1604$, $1508(\mathrm{C}=\mathrm{C}, \mathrm{Ar}), 1282,1027 \mathrm{~cm}^{-1}$

${ }^{1} \mathrm{H}-\mathrm{NMR}$ (DMSO-d6, $\left.300 \mathrm{MHz}\right): \delta 3.84(\mathrm{~s}, 6 \mathrm{H}$, $\left.2 \times \mathrm{OCH}_{3}\right), 6.06(\mathrm{~s}, 1 \mathrm{H}), 6.71(\mathrm{~d}, 2 \mathrm{H}, J=16.6 \mathrm{~Hz})$, $6.82(\mathrm{~d}, 2 \mathrm{H}, J=8.22 \mathrm{~Hz}$, ortho coupling), $7.16(\mathrm{~d}, 2 \mathrm{H}$, $J=8.03 \mathrm{~Hz}$, ortho coupling), $7.33(\mathrm{~s}, 2 \mathrm{H}), 7.55$ (d, $2 \mathrm{H}, J=15.35 \mathrm{~Hz}), 9.74(\mathrm{~s}, 2 \mathrm{H}, 2 \times$ phenolic $\mathrm{OH})$, 10.13 (s, 1H, enol OH) ppm

${ }^{13} \mathrm{C}-\mathrm{NMR}(\mathrm{DMSO}-d 6,75 \mathrm{MHz}): \delta 56.22\left(2 \times \mathrm{OCH}_{3}\right)$, $101.43,111.82,116.23,121.62,123.70,126.86$, $141.28,148.53,149.88,183.76 \mathrm{ppm}$

Mass (EI): $m / z 369\left(\mathrm{MH}^{+}\right)$

\section{(1E,4Z, 6E)-5-hydroxy-1,7-diphenylhepta-}

\section{1,4,6-trien-3-one}<smiles>O=C(/C=C/C(O)=C/c1ccccc1)/C=C/c1ccccc1</smiles> 
IR (KBr): $v$ 3398, 2942，1684， 1605, 1172 , $1025 \mathrm{~cm}^{-1}$

${ }^{1} \mathrm{H}$ NMR (300 MHz, $\left.\mathrm{CDCl}_{3}\right): \delta 6.54(1 \mathrm{H}, \mathrm{s}, \mathrm{H}-4)$, $6.73(1 \mathrm{H}, \mathrm{d}, J=16.0 \mathrm{~Hz}, \mathrm{H}-7), 6.82(1 \mathrm{H}, \mathrm{d}$, $J=16.0 \mathrm{~Hz}, \mathrm{H}-6), 7.15(1 \mathrm{H}, \mathrm{d}, J=16.0 \mathrm{~Hz}, \mathrm{H}-2)$, 7.27 (2H, dd, $\left.J=8.0,2 \mathrm{~Hz}, \mathrm{H}-4^{\prime}, 4^{-\prime}\right), 7.37$ (4H, dd, $\left.J=8.0 \mathrm{~Hz}, \mathrm{H}-3^{\prime}, 5^{\prime}, 3^{\prime \prime}, 5^{-\prime}\right), 7.51(4 \mathrm{H}, \mathrm{dd}, J=8.0$, $\left.2.0 \mathrm{~Hz}, \mathrm{H}-2^{\prime}, 6^{\prime}, 2^{\prime \prime}, 6^{\prime \prime}\right), 7.64(1 \mathrm{H}, \mathrm{d}, J=16.0 \mathrm{~Hz}$, H-1) ppm

${ }^{13} \mathrm{C} \quad \mathrm{NMR} \quad\left(75 \mathrm{MHz}, \quad \mathrm{CDCl}_{3}\right): \quad \delta \quad 101.2 \quad(\mathrm{C}-4)$, 118.3 (C-6), 122.9 (C-2), 126.5 (C-4, $4^{-\prime}$, $128.3\left(\mathrm{C}-2^{-}, 6^{\prime}, 2^{-}, 6^{-}\right), 128.6\left(\mathrm{C}-3^{-}, 5^{\top}, 3^{\prime}\right.$, $\left.5^{-\prime}\right), 135.2$ (C-1', 1"), 140.5 (C-7), 142.3 (C-1), $182.4(\mathrm{C}-3, \mathrm{C}-5) \mathrm{ppm}$

(1E,4Z, 6E)-5-hydroxy-1,7-dip-tolylhepta1,4,6-trien-3-one<smiles>Cc1ccc(/C=C/C(=O)/C=C(O)/C=C/c2ccc(C)cc2)cc1</smiles>

IR (KBr): v 3422， 2942， 1672， 1605， 1172, $1025 \mathrm{~cm}^{-1}$

${ }^{1} \mathrm{H}$ NMR (300 MHz, $\left.\mathrm{CDCl}_{3}\right): \delta 2.35\left(6 \mathrm{H}, \mathrm{s}, \mathrm{ArCH}_{3}\right)$, $6.52(1 \mathrm{H}, \mathrm{s}, \mathrm{H}-4), 6.83(1 \mathrm{H}, \mathrm{d}, J=16.0 \mathrm{~Hz}, \mathrm{H}-7)$, $6.87(1 \mathrm{H}, \mathrm{d}, J=16.0 \mathrm{~Hz}, \mathrm{H}-6), 7.06(1 \mathrm{H}, \mathrm{d}$, $J=16.0 \mathrm{~Hz}, \mathrm{H}-2) 7.13$ (2H, dd, $J=8.0,2 \mathrm{~Hz}, \mathrm{H}-3^{\prime}$, $\left.5^{\prime}, 3^{\prime}, 5^{-\prime}\right), 7.47\left(4 \mathrm{H}, \mathrm{dd}, J=8.0 \mathrm{~Hz}, \mathrm{H}-2^{\prime}, 6^{\prime}, 2^{\prime}\right.$, $\left.6^{-\prime}\right), 7.69(1 \mathrm{H}, \mathrm{d}, J=16.0 \mathrm{~Hz}, \mathrm{H}-1) \mathrm{ppm}$

${ }^{13} \mathrm{C} \quad \mathrm{NMR} \quad\left(75 \mathrm{MHz}, \quad \mathrm{CDCl}_{3}\right): \quad \delta \quad 21.4 \quad\left(\mathrm{CH}_{3}\right)$, 101.1 (C-4), 118.5 (C-6), 123.2 (C-2), 128.4 (C-2', $\left.6^{\prime}, 2^{-\prime}, 6^{\prime \prime}\right), 128.7\left(\mathrm{C}-3^{\prime}, 5^{\prime}, 3^{\prime}, 5^{\prime \prime}\right), 130.9$ (C-4', $\left.4^{\prime \prime}\right), 132.2$ (C-1', 1"), 140.4 (C-7), 142.2 (C-1), $182.2(\mathrm{C}-3, \mathrm{C}-5) \mathrm{ppm}$

(1E,4Z, 6E)-1,7-bis(4-chlorophenyl)5-hydroxyhepta-1,4,6-trien-3-one<smiles>O=C(/C=C/C(O)=C/c1ccc(Cl)cc1)/C=C/c1ccc(Cl)cc1</smiles>

IR (KBr): v 3422，2932，1685，1603，1172, $1025 \mathrm{~cm}^{-1}$

${ }^{1} \mathrm{H}$ NMR (300 MHz, CDCl $)$ ): $\delta 6.57$ (1H,s, H-4), $6.82(1 \mathrm{H}, \mathrm{d}, J=16.0 \mathrm{~Hz}, \mathrm{H}-7), 6.88(1 \mathrm{H}, \mathrm{d}$, $J=16.0 \mathrm{~Hz}, \mathrm{H}-6), 7.08(1 \mathrm{H}, \mathrm{d}, J=16.0 \mathrm{~Hz}, \mathrm{H}-2)$, $7.32\left(2 \mathrm{H}, \mathrm{dd}, J=8.0,2 \mathrm{~Hz}, \mathrm{H}-3^{-}, 5^{-}, 3^{-}, 5^{-\prime}\right)$, $7.52\left(4 \mathrm{H}, \mathrm{dd}, J=8.0 \mathrm{~Hz}, \mathrm{H}-2^{\prime}, 6^{\prime}, 2^{\prime}, 6^{-\prime}\right), 7.71(1 \mathrm{H}$, $\mathrm{d}, J=16.0 \mathrm{~Hz}, \mathrm{H}-1) \mathrm{ppm}$

${ }^{13} \mathrm{C} \quad \mathrm{NMR} \quad\left(75 \mathrm{MHz}, \quad \mathrm{CDCl}_{3}\right): \quad \delta 101.0 \quad(\mathrm{C}-4)$, 118.9 (C-6), 123.5 (C-2), 128.6 (C-3', 5', 3'), $\left.5^{\prime \prime}\right), \quad 129.2$ (C-2', 6', 2', 2'), 131.9 (C-1', $\left.1^{\prime \prime}\right), 133.6$ (C-4', 4") 140.6 (C-7), 142.4 (C-1), $182.5(\mathrm{C}-3, \mathrm{C}-5) \mathrm{ppm}$
(1E,4Z,6E)-1,7-bis(2-chlorophenyl)-

5-hydroxyhepta-1,4,6-trien-3-one<smiles>O=C(/C=C/C(O)=C/c1ccccc1Cl)/C=C/c1ccccc1Cl</smiles>

IR (KBr): v 3374, 2932, 1627, 1683, $1173 \mathrm{~cm}^{-1}$

${ }^{1} \mathrm{H}$ NMR (300 MHz, $\left.\mathrm{CDCl}_{3}\right): \delta 6.61(1 \mathrm{H}, \mathrm{s}, \mathrm{H}-4)$, $6.87(1 \mathrm{H}, \mathrm{d}, J=16.0 \mathrm{~Hz}, \mathrm{H}-6), 6.87(1 \mathrm{H}, \mathrm{d}, J=16 \mathrm{~Hz}$, H-2), 7.09 (1H, d, $J=16 \mathrm{~Hz}, \mathrm{H}-7), 7.28(2 \mathrm{H}, \mathrm{dd}$, $\left.J=8.0,2 \mathrm{~Hz}, \mathrm{H}-4^{\prime}, 4^{-\prime}\right), 7.33\left(2 \mathrm{H}, J=8,2 \mathrm{~Hz}, \mathrm{H}-5^{\prime}\right.$, $\left.5^{\prime \prime}\right), 7.37$ (2H, dd, $\left.J=8,2 \mathrm{~Hz}, \mathrm{H}-6^{\prime}, 6^{\prime \prime}\right), 7.48(2 \mathrm{H}$, $\left.\mathrm{dd}, J=8.0 \mathrm{~Hz}, \mathrm{H}-3^{\prime}, 3^{-\prime}\right), 7.84(2 \mathrm{H}, \mathrm{d}, J=16.0 \mathrm{~Hz}$, $\mathrm{H}-1) \mathrm{ppm}$

${ }^{13} \mathrm{C}$ NMR (75MHz, $\left.\mathrm{CDCl}_{3}\right): \delta 101.5(\mathrm{C}-4), 118.6(\mathrm{C}-6)$, 123.7 (C-2), 127.3 (C-5', 5“), 128.4 (C-6', 6"),

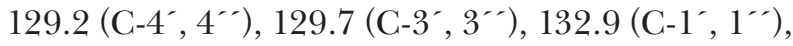
134.6 (C-2', 2'), 140.4 (C-7), 142.7 (C-1), 182.8 (C-3, C-5) ppm

\section{(1E,4Z,6E)-5-hydroxy-}

1,7-bis(4-methoxyphenyl)hepta-1,4,6-trien-3-one<smiles>COc1ccc(/C=C/C(=O)/C=C(O)/C=C/c2ccc(OC)cc2)cc1</smiles>

IR (KBr): v 3441, 2933, 1672, 1600, 1177, 1028, 977, $826 \mathrm{~cm}^{-1}$

${ }^{1} \mathrm{H}$ NMR $\left(300 \mathrm{MHz}, \mathrm{CDCl}_{3}\right): \delta 3.84(6 \mathrm{H}, \mathrm{s}, 2-\mathrm{Ar}-$ $\left.\mathrm{OCH}_{3}\right), 6.74(1 \mathrm{H}, \mathrm{s}, \mathrm{H}-4), 6.82(1 \mathrm{H}, \mathrm{d}, J=16 \mathrm{~Hz}$, $\mathrm{H}-7), 6.92(1 \mathrm{H}, \mathrm{d}, J=16 \mathrm{~Hz}, \mathrm{H}-6), 6.98(4 \mathrm{H}, \mathrm{d}$, $\left.J=8.7 \mathrm{~Hz}, \mathrm{H}-3^{\prime}, 5^{\prime}, 3^{-\prime}, 5^{-\prime}\right), 7.06(1 \mathrm{H}, \mathrm{d}, J=16 \mathrm{~Hz}$, $\mathrm{H}-2), 7.46$ (4H, d, $\left.J=8.7 \mathrm{~Hz}, \mathrm{H}-2^{\prime}, 6^{-}, 2^{-\prime}, 6^{-\prime}\right)$, $7.69(2 \mathrm{H}, d, J=15.7 \mathrm{~Hz}, \mathrm{H}-1)$ ppm

${ }^{13} \mathrm{C}$ NMR $\left(75 \mathrm{MHz}, \mathrm{CDCl}_{3}\right): \delta 56.2\left(\mathrm{OCH}_{3}\right), 101.3$ (C-4), 114.6 (C-3', 5', $\left.3^{\prime \prime}, 5^{\prime \prime}\right), 118.6$ (C-6), 123.5 (C-2), 128.1 (C-1', $\left.1^{\prime \prime}\right), 130.4$ (C-2', 6', 2', $2^{\prime \prime}$ ), 140.6 (C-7), 142.4 (C-1), 159.7 (C-4', $\left.4^{\prime \prime}\right), 182.3$ (C-3, C-5) ppm

(1E,4Z, 6E)-5-hydroxy-

1,7-bis(2-hydroxyphenyl)hepta-1,4,6-trien-3-one<smiles>O=C(/C=C(O)/C=C/c1ccccc1O)/C=C/c1ccccc1O</smiles>

IR (KBr): v 3412, 3391, 1615, 1255, 1142, 961, $752 \mathrm{~cm}^{-1}$

${ }^{1} \mathrm{H}$ NMR (300 MHz, DMSO-d6): $\delta 6.16$ (1H, s, H-4), $6.64(1 \mathrm{H}, \mathrm{d}, J=16 \mathrm{~Hz}, \mathrm{H}-6), 6.87(2 \mathrm{H}, \mathrm{d}, J=7.5 \mathrm{~Hz}$, H-3', $\left.3^{-\prime}\right), 6.95$ (2H, d, J = $\left.16 \mathrm{~Hz}, \mathrm{H}-2\right), 7.05(1 \mathrm{H}$, $\mathrm{d}, J=16 \mathrm{~Hz}, \mathrm{H}-7), 7.25\left(4 \mathrm{H}, \mathrm{m}, \mathrm{H}-4^{\prime}, 5^{\prime}, 4^{\prime \prime}, 5^{-\prime}\right)$, $7.67\left(2 \mathrm{H}, \mathrm{d}, J=7.5 \mathrm{~Hz}, \mathrm{H}-6^{\prime}, 6^{\prime \prime}\right), 7.89$ (1H, d, $J=16 \mathrm{~Hz}, \mathrm{H}-1) \mathrm{ppm}$ 
${ }^{13} \mathrm{C}$ NMR (75 MHz, DMSO- $\left.d 6\right): \delta 101.3$ (C-4), 117.2 (C-3', 3“), 118.9 (C-6), 121.1 (C-5', 5“), 122.2 (C-1', 1'”), 122.9 (C-2), 127.3 (C-5', 5 '), 128.8 (C-6', 6), 129.4 (C-4', 4), 140.1 (C-7), 142.6 (C-1), 157.3 (C-2`, 2־), 182.4 (C-3, C-5) ppm

(1E,4Z,6E)-1,7-bis(3,4-dimethoxyphenyl)-

5-hydroxyhepta-1,4, 6-trien-3-one<smiles>COc1ccc(/C=C/C(=O)/C=C(O)/C=C/c2ccc(OC)c(OC)c2)cc1OC</smiles>

IR (KBr): v 3420, 2933, 1676, 1592, 1177, 1028, 977, $826 \mathrm{~cm}^{-1}$

${ }^{1} \mathrm{H}$ NMR (300 MHz, $\left.\mathrm{CDCl}_{3}\right): \delta 3.92,(\mathrm{~s}, 6 \mathrm{H}), 3.97$ (s, $6 \mathrm{H}), 6.67$ (1H, s, H-4), 6.83 (d, J=16.0 Hz, 1H, H-7), $6.89(1 \mathrm{H}, \mathrm{d}, J=16 \mathrm{~Hz}, \mathrm{H}-6), 6.97(2 \mathrm{H}, \mathrm{d}, J=8.0 \mathrm{~Hz}$, H-5 , $\left.5^{-\prime}\right), 7.05$ (1H, d, J=16Hz, H-2), 7.13 (2H, d, $\left.J=2.0 \mathrm{~Hz}, \mathrm{H}-2^{\prime}, 2^{-\prime}\right), 7.21(2 \mathrm{H}, \mathrm{dd}, J=2.0,8.0 \mathrm{~Hz}$, H-6', 6“-), 7.68 (2H, d, J=16.0 Hz, H-1) ppm ${ }^{13} \mathrm{C}$ NMR $\left(75 \mathrm{MHz}, \quad \mathrm{CDCl}_{3}\right): \delta \quad 55.8 \quad\left(\mathrm{OCH}_{3}\right)$, $56.2\left(\mathrm{OCH}_{3}\right), 101.5(\mathrm{C}-4), 111.9\left(\mathrm{C}-2^{\prime}, 2^{\prime-}\right), 112.3\left(\mathrm{C}-5^{\prime}\right.$, $\left.5^{\prime-}\right), 118.4$ (C-6), 122.4 (C-6', 6'), 123.2 (C-2), $127.4\left(\mathrm{C}^{\prime} 1^{\prime}, 1^{\prime \prime}\right), 140.3$ (C-7), 142.6 (C-1), 149.0 (C-4', $\left.4^{-\prime}\right), 149.7$ (C-3', 3') $182.8(\mathrm{C}-3, \mathrm{C}-5)$ ppm

(1E,4Z,6E)-5-hydroxy-

1,7-bis(4-nitrophenyl)hepta-1,4,6-trien-3-one<smiles>O=C(/C=C/C(O)=C/c1ccc([N+](=O)[O-])cc1)/C=C/c1ccc([N+](=O)[O-])cc1</smiles>

IR (KBr): v 3452, 2933, 1692, 1608, 1177, 1028, $977,826 \mathrm{~cm}^{-1}$

${ }^{1} \mathrm{H}$ NMR (300 MHz, $\left.\mathrm{CDCl}_{3}\right): \delta 6.83(1 \mathrm{H}, \mathrm{s}, \mathrm{H}-4)$, 6.91(1H, d, J=16 Hz, H-7), $7.08(2 \mathrm{H}, \mathrm{d}, J=15.7 \mathrm{~Hz}$, $\mathrm{H}-, 6), 7.28(1 \mathrm{H}, \mathrm{d}, J=16 \mathrm{~Hz}, \mathrm{H}-2), 7.92(2 \mathrm{H}, \mathrm{d}$, $J=15.7 \mathrm{~Hz}, \mathrm{H}-1), 8.12\left(4 \mathrm{H}, \mathrm{d}, J=8.7 \mathrm{~Hz}, \mathrm{H}^{\prime} 2^{\prime}, 6^{\prime}\right.$, $\left.2^{\prime \prime}, 6^{-\prime}\right), 7.98\left(4 \mathrm{H}, \mathrm{d}, J=8.7 \mathrm{~Hz}, \mathrm{H}-3^{\prime}, 5^{\prime}, 3^{\prime \prime}, 5^{\prime \prime}\right)$ ppm

${ }^{13} \mathrm{C} \mathrm{NMR}\left(75 \mathrm{MHz}, \mathrm{CDCl}_{3}\right): \delta 101.8(\mathrm{C}-4), 118.8(\mathrm{C}-6)$,

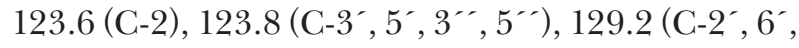
$\left.2^{\prime \prime}, 2^{\prime \prime}\right), 140.6$ (C-7), $141.3\left(\mathrm{C}^{\prime} 1^{\prime}, 1^{\prime \prime}\right), 142.6$ (C-1), $147.4\left(\mathrm{C}^{-} 4^{\prime}, 4^{-\prime}\right), 182.8$ (C-3, C-5) ppm

\section{(1E,4Z,6E)-5-hydroxy-}

1,7-bis(3-nitrophenyl)hepta-1,4,6-trien-3-one<smiles>O=C(/C=C/c1cccc([N+](=O)[O-])c1)/C=C/c1cccc([N+](=O)[O-])c1</smiles>

IR (KBr): v 3425, 2933, 1695, 1608, 1177, 1028, $977,826 \mathrm{~cm}^{-1}$

${ }^{1} \mathrm{H}$ NMR (300 $\left.\mathrm{MHz}, \mathrm{CDCl}_{3}\right): \delta 6.78(1 \mathrm{H}, \mathrm{s}$, H-4), 6.86 (1H, d, J = $16 \mathrm{~Hz}, \mathrm{H}-7), 7.12(1 \mathrm{H}, \mathrm{d}$, $J=16 \mathrm{~Hz}, \mathrm{H}-6), 7.23(2 \mathrm{H}, \mathrm{d}, J=15.7 \mathrm{~Hz}, \mathrm{H}-2)$, $7.58\left(2 \mathrm{H}, \mathrm{dd}, J=8,8 \mathrm{~Hz}, \mathrm{H}-5^{\prime}, 5^{-\prime}\right), 7.95(1 \mathrm{H}, \mathrm{d}$, $J=15.7 \mathrm{~Hz}, \mathrm{H}-1), 7.98\left(2 \mathrm{H}, \mathrm{dd}, J=8,2 \mathrm{~Hz}, \mathrm{H}-6^{\prime}\right.$, $\left.6^{\prime \prime}\right), 8.06$ (2H, m, H-4`, $\left.4^{\prime \prime}\right), 8.16(2 \mathrm{H}, \mathrm{d}, J=2 \mathrm{~Hz}$, $\left.\mathrm{H}-2^{\prime}, 2^{-\prime}\right) \mathrm{ppm}$

${ }^{13} \mathrm{C} \mathrm{NMR}\left(75 \mathrm{MHz}, \mathrm{CDCl}_{3}\right)$ : $\delta 101.4(\mathrm{C}-4), 119.2(\mathrm{C}-6)$, 122.7 (C-2', 2-) 123.1 (C-2), 123.4 (C-4', 4"), $129.4\left(\mathrm{C}^{-} 5^{\prime}, 5^{\prime-}\right), 134.3\left(\mathrm{C}^{\prime} 6^{\prime}, 6^{\prime \prime}\right), 137.5\left(\mathrm{C}^{\prime} 1^{\prime}, 1^{\prime \prime}\right)$, 139.9 (C-7), 142.3 (C-1), 147.4 (C-3', $3^{-\prime}$ ), 182.4 (C-3, C-5) ppm

\section{$(1 E, 4 Z, 6 E)-5-h y d r o x y$ -}

1,7-bis(2-nitrophenyl)hepta-1,4,6-trien-3-one<smiles>O=C(/C=C/C(O)=C/c1ccccc1[N+](=O)[O-])/C=C/c1ccccc1[N+](=O)[O-]</smiles>

IR (KBr): v 3455, 1697, 1596, 1255, 1142, 961, $752 \mathrm{~cm}^{-1}$

${ }^{1} \mathrm{H}$ NMR (300 MHz, $\left.\mathrm{CDCl}_{3}\right): \delta 6.78(1 \mathrm{H}, \mathrm{s}, \mathrm{H}-4)$, $6.84(1 \mathrm{H}, \mathrm{d}, J=16 \mathrm{~Hz}, \mathrm{H}-6), 7.10(1 \mathrm{H}, \mathrm{d}, J=16 \mathrm{~Hz}$, H-2), 7.32 (1H, d, J=16 Hz, H-7), 7.73 (2H, m, H-5', $\left.5^{\prime-}\right), 7.91\left(2 \mathrm{H}, \mathrm{m}, \mathrm{H}-4^{\prime}, 4^{-\prime}\right), 8.02(2 \mathrm{H}, \mathrm{dd}, J=8$, $\left.2 \mathrm{~Hz}, \mathrm{H}-6^{\prime}, 6^{\prime \prime}\right), 8.14$ (2H, d, J=8, $\left.2 \mathrm{~Hz}, \mathrm{H}-3^{\prime}, 3^{-\prime}\right)$, $8.27(2 \mathrm{H}, \mathrm{d}, J=15.8 \mathrm{~Hz}, \mathrm{H}-1,7) \mathrm{ppm}$

${ }^{13} \mathrm{C}$ NMR (75 MHz, $\mathrm{CDCl}_{3}$ ): $\delta 101.2(\mathrm{C}-4), 119.4(\mathrm{C}-6)$, 123.4 (C-2), 123.4 (C-3, 3"'), 127.2 (C-6", 6"), $127.4\left(\mathrm{C}-1^{\prime}, 1^{\prime \prime}\right), 128.9\left(\mathrm{C}-4^{\prime}, 4^{\prime \prime}\right), 134.6\left(\mathrm{C}-5^{\prime}, 5^{-\prime}\right)$, 140.1 (C-7), 142.9 (C-1), 147.5 (C-2, $2^{\prime-}$ ), 182.5 (C-3, C-5) ppm

\section{(1E,4Z,6E)-1,7-bis(4-(dimethylamino)phenyl)- 5-hydroxyhepta-1,4,6-trien-3-one}<smiles>CN(C)c1ccc(/C=C/C(=O)/C=C(O)/C=C/c2ccc(N(C)C)cc2)cc1</smiles>

IR (KBr): v 3417, 2920, 1664, 1362, 962, $814 \mathrm{~cm}^{-1}$ ${ }^{1} \mathrm{H}$ NMR (300 MHz, $\left.\mathrm{CDCl}_{3}\right)$ : $\delta 3.03(12 \mathrm{H}, \mathrm{s}, 2 \cdot \mathrm{Ar}-$ $\left.\mathrm{N}\left(\mathrm{CH}_{3}\right)_{2}\right), 6.25(1 \mathrm{H}, \mathrm{s}, \mathrm{H}-4), 6.72(4 \mathrm{H}, \mathrm{d}, J=8.7 \mathrm{~Hz}$, $\left.\mathrm{H}-3^{-}, 5^{-}, 3^{-}, 5^{-\prime}\right), 6.84(1 \mathrm{H}, \mathrm{d}, J=16 \mathrm{~Hz}, \mathrm{H}-7)$, $6.91(1 \mathrm{H}, \mathrm{d}, J=16 \mathrm{~Hz}, \mathrm{H}-6), 7.05(1 \mathrm{H}, \mathrm{d}, J=16 \mathrm{~Hz}$, H-2), 7.42 (4H, d, $\left.J=8.7 \mathrm{~Hz}, \mathrm{H}^{\prime} 2^{\prime}, 6^{\prime}, 2^{\prime \prime}, 6^{\prime \prime}\right)$, $7.63(2 \mathrm{H}, \mathrm{d}, J=16 \mathrm{~Hz}, \mathrm{H}-1)$

${ }^{13} \mathrm{C}$ NMR $\left(75 \mathrm{MHz}, \mathrm{CDCl}_{3}\right): \delta 42.4 \quad\left(\mathrm{~N}\left(\mathrm{CH}_{3}\right)_{2}\right)$, 101.6 (C-4), 110.9 (C-3', 5', 3"', 5'), 118.9 (C-6), 123.4 (C-2), 124.3 (C-1', $\left.1^{\prime \prime}\right), 129.6$ (C-2', 6', 2'”, 2“), 140.3 (C-7), 142.1 (C-1), 149.7 (C-4', 4“), $182.6(\mathrm{C}-3, \mathrm{C}-5)$ 
(1E,4Z,6E)-1,7-bis(4-bromophenyl)-

5-hydroxyhepta-1,4,6-trien-3-one<smiles>O=C(/C=C(O)/C=C/c1ccc(Br)cc1)/C=C/c1ccc(Br)cc1</smiles>

IR (KBr): v 3412, 2932, 1627, 1603, 962, $814 \mathrm{~cm}^{-1}$ ${ }^{1} \mathrm{H}$ NMR $\left(300 \mathrm{MHz}_{\mathrm{CDCl}}\right): \delta 6.52(1 \mathrm{H}, \mathrm{s}, \mathrm{H}-4)$, $6.82(1 \mathrm{H}, \mathrm{d}, J=16.0 \mathrm{~Hz}, \mathrm{H}-7), 6.89(1 \mathrm{H}, \mathrm{d}, J$ $=16 \mathrm{~Hz}, \mathrm{H}-6), 7.04(1 \mathrm{H}, \mathrm{d}, J=16 \mathrm{~Hz}, \mathrm{H}-2), 7.35(2 \mathrm{H}$, $\left.\mathrm{dd}, J=8.0,2 \mathrm{~Hz}, \mathrm{H}-3^{\prime}, 5^{\prime}, 3^{-}, 5^{-\prime}\right), 7.56(4 \mathrm{H}, \mathrm{dd}$, $\left.J=8.0 \mathrm{~Hz}, \mathrm{H}^{-} 2^{\prime}, 6^{\prime}, 2^{\prime}, 6^{-\prime}\right), 7.75(1 \mathrm{H}, \mathrm{d}, J=16.0 \mathrm{~Hz}$, H-1) ppm

${ }^{13} \mathrm{C} \mathrm{NMR}\left(75 \mathrm{MHz}, \mathrm{CDCl}_{3}\right): \delta 101.1$ (C-4), 118.6 (C-6), 122.6 (C-4', 4'), 123.1 (C-2), 128.6 (C-2', 6', 2'”, $\left.2^{-\prime}\right), 130.6\left(\mathrm{C}^{-3} 3^{\prime}, 5^{\prime}, 3^{\prime \prime}, 5^{\prime \prime}\right), 131.9$ (C-1', 1“), 140.4 (C-7), 142.2 (C-1), 182.5 (C-3, C-5) ppm

(1E, 4Z, 6E)-5-hydroxy-

1,7-bis(4-hydroxyphenyl)hepta-1,4,6-trien-3-one<smiles>O=C(/C=C(O)/C=C/c1ccc(O)cc1)/C=C/c1ccc(O)cc1</smiles>

IR (KBr): v 3412, 3211, 1620, 1600, 1269, 1168, $1140,955,831 \mathrm{~cm}^{-1}$

${ }^{1} \mathrm{H}$ NMR (300 MHz, DMSO- $\left.d 6\right): \delta 6.12(1 \mathrm{H}, \mathrm{s}$, $\mathrm{H}-4), 6.73(1 \mathrm{H}, \mathrm{d}, J=16.0 \mathrm{~Hz}, \mathrm{H}-7), 6.83(1 \mathrm{H}, \mathrm{d}$, $J=16 \mathrm{~Hz}, \mathrm{H}-6), 6.87\left(4 \mathrm{H} \mathrm{d}, J=8.0 \mathrm{~Hz}, \mathrm{H}-3^{\prime}, 5^{\prime}\right.$, $\left.3^{\prime \prime}, 5^{\prime \prime}\right), 7.56\left(4 \mathrm{H}, \mathrm{d}, J=8.0 \mathrm{~Hz}, \mathrm{H}-2^{\prime}, 6^{\prime}, 2^{\prime \prime}, 6^{\prime \prime}\right)$, $7.67(2 \mathrm{H}, \mathrm{d}, J=16.0 \mathrm{~Hz}, \mathrm{H}-1) \mathrm{ppm}$

${ }^{13} \mathrm{C}$ NMR (75 MHz, DMSO-d6): $\delta 101.1$ (C-4), $115.3\left(\mathrm{C}-3^{\prime}, 5^{\prime}, 3^{\cdots}, 5^{\prime}\right), 118.3$ (C-6), 123.2 (C-2), $127.4\left(\mathrm{C}^{\prime} 1^{\prime}, 1^{\prime \prime}\right), 130.6\left(\mathrm{C}-2^{\prime}, 6^{\prime}, 2^{\prime \prime}, 2^{\prime \prime}\right), 140.4(\mathrm{C}-7)$, 142.7 (C-1), 159.7 (C-4', $\left.4^{\prime \prime}\right), 182.6$ (C-3, C-5) ppm

(1E,4Z,6E)-5-hydroxy-

1,7-bis(2-methoxyphenyl)hepta-1,4,6-trien-3-one<smiles>COc1ccccc1/C=C/C(=O)/C=C(O)/C=C/c1ccccc1OC</smiles>

IR (KBr): $v$ 3412, 1668, 1255, 1142, 961, $752 \mathrm{~cm}^{-1}$ ${ }^{1} \mathrm{H}$ NMR (300 MHz, $\left.\mathrm{CDCl}_{3}\right): \delta 3.85(6 \mathrm{H}, \mathrm{s}, 2 \cdot \mathrm{Ar}-$ $\left.\mathrm{OCH}_{3}\right), 6.13(1 \mathrm{H}, \mathrm{s}, \mathrm{H}-4), 6.62(1 \mathrm{H}, \mathrm{d}, J=16 \mathrm{~Hz}$, H-6), 6.79 (2H, d, J = 7.5 Hz, H-3', 3') $6.83(1 \mathrm{H}$, $\mathrm{d}, J=16 \mathrm{~Hz}, \mathrm{H}-2), \mathrm{H}-6.97(1 \mathrm{H}, \mathrm{d}, J=16 \mathrm{~Hz}$, $\left.\mathrm{H}^{-7}\right), 7.17$ (4H, m, H-4, 5', $\left.4^{\prime \prime}, 5^{\prime \prime}\right), 7.53$ (2H, d, $\left.J=7.5 \mathrm{~Hz}, \mathrm{H}-6^{\prime}, 6^{-\prime}\right), 7.74(2 \mathrm{H}, \mathrm{d}, J=16 \mathrm{~Hz}, \mathrm{H}-1)$ ppm

${ }^{13} \mathrm{C} \quad \mathrm{NMR} \quad\left(75 \mathrm{MHz}, \quad \mathrm{CDCl}_{3}\right): \delta \quad 55.4 \quad\left(\mathrm{OCH}_{3}\right)$, 101.2 (C-4), 115.2 (C-3', 3') 118.5 (C-6), 120.8 (C-5', $\left.5^{\prime \prime}\right), 122.9$ (C-2), 127.9 (C-4', $\left.4^{\prime \prime}\right), 125.4$ (C-1',

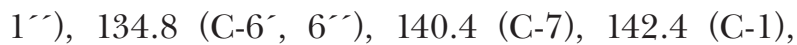
159.3 (C-2', 2') 182.4 (C-3, C-5) ppm

(1E,4Z,6E)-1,7-di(anthracen-9-yl)-

5-hydroxyhepta-1,4,6-trien-3-one<smiles>O=C(/C=C/C(O)=C/c1c2ccccc2cc2ccccc12)/C=C/c1c2ccccc2cc2ccccc12</smiles>

IR $(\mathrm{KBr}):$ v 3415, 1682, 1594, 1255, 1142, 961, $752 \mathrm{~cm}^{-1}$

${ }^{1} \mathrm{H}$ NMR (300 MHz, $\left.\mathrm{CDCl}_{3}\right): \delta 6.65(1 \mathrm{H}, \mathrm{s}, \mathrm{H}-4)$, $6.74(1 \mathrm{H}, \mathrm{d}, J=16 \mathrm{~Hz}, \mathrm{H}-7), 6.86(1 \mathrm{H}, \mathrm{d}, J=16 \mathrm{~Hz}$, H-6), 7.06 (1H, d, J=16 Hz, H-2), 7.39 (8H, m, H-2, $\left.3,6,7,2^{-}, 3^{-}, 6^{-}, 7^{-\prime}\right), 7.53(2 \mathrm{H}, \mathrm{d}, J=7.5 \mathrm{~Hz}$, H-6', 6“-), 7.83 (1H, d, J = $16 \mathrm{~Hz}, \mathrm{H}-1), 7.93$ (8H, m, H-1, 4, 5, 8, 1" $\left., 4^{\prime \prime}, 5^{\prime \prime}, 8^{\prime \prime}\right), 8.12$ (2H, s, H-10, $\left.10^{-\prime}\right) \mathrm{ppm}$

${ }^{13} \mathrm{C}$ NMR $\left(75 \mathrm{MHz}, \mathrm{CDCl}_{3}\right): \delta 101.2(\mathrm{C}-4), 118.9$ (C-6), 121.3 (C-10, 10), 123.1 (C-2), 125.6 (C-3, 6, 3', $\left.6^{\prime \prime}\right), 125.8$ (C-2, 7, 2“, $\left.7^{\prime \prime}\right), 125.5\left(\mathrm{C}-1,8,1^{\prime \prime}, 8^{\cdots}\right)$,

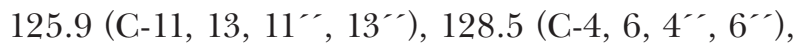
131.3 (C-12, 14, 12“, 14), 131.5 (C-7), 133.4 (C-9, $\left.9^{\prime \prime}\right), 152.8$ (C-1), 182.8 (C-3,5) ppm

\section{Conclusion}

In conclusion, the present paper describes a facile route for one-pot synthesis of curcumin and its analogs using cheap calcium hydroxide as mild base. Compared to literature methods, the present method is remarkable in terms of reaction conditions, work up procedure and high yields of the product obtained and the method would find wide spread application in synthesis of potent curcumin analogs of therapeutic significance.

\section{Acknowledgement}

Dr. Ravi Varala thanks Prof. Rajendra Sahu (Director, IIIT Basar) and Prof. R. V. Raja Kumar (Vice Chancellor, RGUKT) for their support.

\section{References}

Anand P, Thomas SG, Kunnumakkara AB, Sundarm C, Misra K, Priyadarsini IK, Rajasekharan KN, Aggarwal BB (2008) Biochem. Pharm.76: 1590-1611.

Babua KVD, Rajasekharan KN (1994) Organic Preparations and Procedures International: The New Journal for Organic Synthesis 26: 674-677.

Bratu MG (2004) Coloranti alimentari polifunctionali, Teza de doctorat (parte experimentala).

Bratu MG (2005) Acta Universitatis Cibiniensis Series E: Food Technology 9: 11-17. 
Chakraborty S, Ghosh U, Bhattacharyya NP (2006) Mutat. Res. 596: 81-90.

Chen M-Y, Wang J-X, Chen K-T, Wen B-Z, Lin W-C, Chen C-C (2011) J. Chin. Chem. Soc., 58: 1-6.

Elavarasan S, Bhakiaraj D, Chellakili B, Elavarasan T, Gopalakrishnan M (2012) Spectrochimica Acta Part A: Molecular and Biomolecular Spectroscopy 97: 717-721.

Furness MS, Robinson TP, Ehlers T, Hubbard IV RB, Arbiser JL, Goldsmith DJ, Bowen JP (2005) Curr. Pharm. Des., 11: 357-373.

Greenwood NN, Earnshaw A (1984). In: Chemistry of the elements, (pp 117-154). Pergamon Press, New York.

Hahm ER, Cheon G, Lee JH, Kim BJ, Park CH, Yang CH (2002) Cancer Lett. 184: 89-96.

Hari SG, Lee YR (2011) Bull. Korean Chem. Soc. 32: 2949-2954.

Hsu CH, Cheng AL (2007) Adv. Exp. Med. Biol. 595: 471-480.

Jagetia GC, Aggarwal BB (2007) J. Clin. Immunology 27: $19-35$.

Jensen BS (1959) Acta. Chem. Scand. 13: 1668-1670.

Kumar AP, Aggarwal BB, Bharti AC (2003) Anticancer Res. 23: 363-398.

Kuttan R, Sudheeran PC, Josph CD (1985) Cancer Lett. 29: 197-202.
Kuttan R, Sudheeran PC, Josph CD (1987) Tumori 73: 29-31.

Mukherjee N, Chakraborty S, Ghosh U, Bhattacharyya NP, Bhattacharyya RK, Dey S, Roy M (2007) Mol. Cell. Biochem. 297: 31-39.

Liang G, Shao L, Wang Y, Zhao C, Chu Y, Xiao J, Zhao Y, Li X, Yang S (2009) Bioorg. Med. Chem. 17: 2623-2631.

Narayana KR, Varala R, Zubaidha PK (2012) Int. J. Org. Chem. 2: 3A., 287-294 and references cited theirin.

Nichols CE, Youssef D, Harris RG, Jha A (2006) Arkivoc (xiii): 64-72.

Nurfina AN, Reksohadiprodjo MS, Timmerman H, Jenie UA, Sugiyanto D, Van Der Goot H (1997) Eur. J. Med. Chem. 32: 321-328.

Paban HJJ (1937) Rec. Trav. Chem. Pays Bas. 83: $167-172$.

Saimoto H, Yoshida K, Murakami T, Morimoto M, Sashiwa H, Shigemasa Y. (1996) J. Org. Chem. 61: 6768-6769.

Tarabasanu C, Gorduza V, Radu F, Mazgareanu M. (1997). In: Coloranti organici de interes alimentar, cosmetic si farmaceutic, Ed. Uni-Pres, Bucuresti. 